\title{
Analysis of the Association Between Polymorphisms within PA/-1 and $A C E$ genes and Ischemic Stroke Outcome After rt-PA Therapy
}

\author{
Marija Dusanovic Pjevic ${ }^{1}$, Ljiljana Beslac Bumbasirevic ${ }^{2}$, Ljubica Vojvodic ${ }^{3}$, Milka Grk ${ }^{1}$, Nela Maksimovic ${ }^{1}$, Tatjana \\ Damnjanovic ${ }^{1}$, Ivana Novakovic ${ }^{1}$, Katarina Kacar ${ }^{3}$, Milica Pesic ${ }^{1}$, Dijana Perovic ${ }^{1}$, Milan Savic ${ }^{3}$, Veljko Maksic ${ }^{3}$, Jelena \\ Trickovic $^{3}$ and Biljana Jekic ${ }^{1}$. \\ ${ }^{1}$ Institute of Human Genetics, Faculty of Medicine, University of Belgrade, Serbia. ${ }^{2}$ Neurology Clinic, Clinical Center \\ of Serbia, Belgrade, Serbia; Faculty of Medicine, University of Belgrade, Serbia. ${ }^{3}$ St. Sava Hospital, Belgrade, Serbia.
}

Received, December 28, 2018; Revised, April 11, 2019; Accepted, April 16, 2019; Published, April 18, 2019.

\begin{abstract}
Purpose: Treatment of Ischemic stroke (IS) in acute phase is based on the use of thrombolytic rt-PA therapy. We aimed to determine whether different alleles and genotypes of $I / D A C E$ gene and $4 G / 5 G$ PAI-1 gene polymorphisms may influence outcome of rt-PA therapy in patients with IS and the occurrence of haemorrhagic transformation (HT). Methods: Our study included 94 consecutive patients with IS treated with rt-PA. Modified Rankin Scale (mRS) at $3{ }^{\text {rd }}$ month after IS was used to determine the stroke outcome, with scores 0-1 defining the favourable outcome, and scores 2-6 defining poor outcome. Genotypisation of the $A C E-1$ I/D polymorphism was performed by polymerase chain reaction and of the $P A I-1 \quad 4 G / 5 G$ polymorphism by polymerase chain reaction - restriction fragment length analysis. Results: Regarding $P A I-I$ $4 G / 5 G$ polymorphism, 44 patients $(46.8 \%)$ were heterozygotes, and the number of $4 G / 4 G$ and $5 G / 5 G$ homozygotes was the same -25 each $(26.6 \%)$. Number of heterozygotes for the $A C E I / D$ polymorphism was $54(57.4 \%), 9$ patients $(9.6 \%)$ had $I I$, and $31(33 \%) D D$ genotypes. A favourable outcome was recorded in 26 (28.0\%) and the poor outcome in $67(72.0 \%)$ patients. Favourable and poor outcome groups did not differ significantly in $P A I-1 \quad 4 G / 5 G$ and $A C E I / D$ polymorphisms genotype or allele frequencies. There was a statistically significant difference in the occurrence of HT between patients with $A C E I I$ and patients with $A C E$ $I D$ or $D D$ genotypes ( $\mathrm{p}=0.035$ ). Conclusion: Results of our study suggest that stroke patients with $A C E I I$ genotype, treated with rt-PA, may be at risk of HT.
\end{abstract}

\section{INTRODUCTION}

Ischemic stroke (IS) occurs due to an embolus or thrombosis causing vascular occlusion in situ in certain brain parts (1). IS is the third leading cause of death (2) and the leading cause of severe disability in the world (3). Around $85-90 \%$ of all stroke cases account for IS. Non-modifiable risk factors for stroke include gender, age, race, ethnicity and different genetic factors. Yet, diabetes mellitus (DM), hypertension, atrial fibrillation (AF), smoking, etc., are known as preventable or modifiable risk factors (5). These risk factors contribute not only to the development of IS (5) but also to poor recovery after IS (6). From the therapeutic point of view, the central zone of ischemia is irrelevant, since nerve cells cannot be saved. However, it is surrounded by penumbra, an area where the neurons are functionally disturbed, but structurally intact, with the possibility for the recovery of their function. Without timely recanalization, an irreversible structural and functional damage in the zone of penumbra will occur. This has a significant effect on neurological damage, that is, on disability level (7).
The therapy of choice in the acute phase of IS is an intravenous administration of recombined tissue plasminogen activator (rt-PA). Tissue plasminogen activator (t-PA) is synthesized mainly in vascular endothelial cells and has a crucial role in fibrinolysis or blood clot dissolving (8). Rt-PA is the only licensed substance until now, that is recommended to be applied during the first 3.5 to $4 \mathrm{~h}$ after IS (9) considering that its function is achieved through blood clot dissolution and vascular recanalization (10). Studies have shown that an early application of this drug leads to a significant functional improvement and better recovery of patients with an IS. However, given that this therapy can cause potentially serious side effects, mostly haemorrhagic complications, the administration criteria are strictly defined (11). Nevertheless, it is still impossible to predict individual response to the rt-PA therapy. Pharmacogenetics studies should enable better understanding of the interpatients' variability.

Corresponding Author: Biljana Jekic,Visegradska 26. Str., Belgrade, Serbia, jekic.b@gmail.com 
At this point, this field of the research is at the beginning, with available results from only several studies, including this one.

Angiotensin I-converting enzyme (ACE) is a significant enzyme in renin-angiotensinaldosterone system and kinin-kallikrein system (12). ACE has a role in vascular remodelling, arteriosclerosis and pathogenesis of hypertension and IS (13). The insertion/deletion polymorphism (I/D) in the $16^{\text {th }}$ intron of the $A C E$ gene refers to the presence of the insertion allele (I) with Alu sequence of $287 \mathrm{bp}$, and the deletion allele (D) without Alu sequence (14). According to some studies, subjects with DD genotype have an increased plasma ACE activity compared to subjects with II genotype (15).

The product of PAI-1 gene (plasminogen activator inhibitor 1) binds to t-PA, forming an inactive complex. In this way, PAI-1 acts as an inhibitor of endogenous fibrinolytic activity (16). Through this mechanism PAI-1 may have influence on rt-PA activity. Insertion-deletion polymorphism in the promoter region of $P A I-1$ gene $(4 \mathrm{G} / 5 \mathrm{G})$ is associated with the plasma level of PAI-1. 4G allele is linked to the higher expression of $P A I-1$ gene, compared to $5 \mathrm{G}$ variant (17).

In this study we have analyzed whether different alleles and genotypes od I/D $A C E$ and 4G/5G $P A I-1$ gene polymorphisms could influence outcome of rt-PA therapy in patients with IS, as well as the occurrence of haemorrhagic transformation, as the most disturbing side effect of the rt-PA therapy.

\section{METHODS}

\section{Patients and data}

This study involved 94 consecutive patients with IS that were treated with rt-PA therapy in Special Hospital of Cerebrovascular diseases "Sveti Sava", Belgrade, in the period of August 2016 to December 2017. Study included patients older than 18 years who were diagnosed with IS and who received rt-PA therapy. Patients with cerebral infarction caused by subarachnoid haemorrhage and sinus venous thrombosis, as well as those who did not give written informed consent to participate, were excluded from the study.

All patients were assessed using Modified Rankin Scale (mRS) 3 months after IS. Using $\mathrm{mRS}$, we determined the favorable outcome as score 0 or 1 and poor outcome as scores 2 to 6 three months after IS, according to literature (18). According to these scores, patients were divided into two groups, favourable and poor outcome. The neurological deficit in all patients was also evaluated using The National Institutes of Health Stroke Scale (NIHSS) at hospital admission, 24 hours after rt-PA therapy and at discharge.

Data on demographic characteristics (age, gender), as well as on clinical characteristics (vascular risk factors - hypertension, atrial fibrillation, diabetes mellitus, ischemic heart disease, heart failure, peripheral artery disease, smoking habit, personal history of diseases) were obtained from medical records of patients at baseline. Custom blood and biochemical tests were performed for all patients at admission. All patients received thrombolytic therapy within 4 hours after onset of acute IS.

Haemorrhagic transformation after rt-PA therapy in CNS was recorded according to European Cooperative Acute Stroke Study (ECASS) III definition (intracranial bleeding on $\mathrm{CT} /$ magnetic resonance imaging with a neurological deterioration of at least 4 points on the NIHSS score from baseline) (19).

Side effects such as bleedings outside of CNS, allergic reactions to medication, epileptic seizures, agitation as well as intrahospital infection was also recorded. After 3 months all consecutive participants were contacted and $\mathrm{mRS}$ evaluation was performed by the specialist of neurology.

The study was approved by the Ethical Committee of the Special Hospital of Cerebrovascular diseases "Sveti Sava" and by the Ethical Commission of the Faculty of Medicine, University of Belgrade.

\section{Molecular genetic analysis}

Total genomic DNA was extracted from peripheral blood leukocytes using a salting out method (20). PCR method was used for the analysis of $A C E-1$ $I / D$ polymorphism, and polymerase chain reactionrestriction fragment length polymorphism (PCRRLP) method for PAI-1 $4 G / 5 G$ polymorphism.

Primers used in PCR reaction for analysis of $A C E-1$ I/D polymorphism were: Fw 5'-CTG GAC ACC ACT CCC ATC CTT TCT -3', and Rev 5'CTG GAC ACC ACT CCC ATC CTT TCT -3'. Conditions for the amplification were as follows: the initial denaturation at $95^{\circ} \mathrm{C}$, in 35 3-stepcycles: a 1-minute denaturation at $94^{\circ} \mathrm{C}$, hybridization at $63^{\circ} \mathrm{C}$ for 30 seconds, primer extension and DNA polymerization at $68^{\circ} \mathrm{C}$ for 1 minute and a final extension at $68^{\circ} \mathrm{C}$ for 7 minutes. Within the amplified sequence of $A C E-1$ gene that was $490 \mathrm{bp}$ long, there was an Alu sequence insertion that corresponds to the I allele. The 
missing Alu sequence corresponds to D allele and 203bp long PCR product.

Analysis for $P A I-14 G / 5 G$ polymorphism was conducted using PCR-RFLP method. Primers that were used in this reaction were constraining a fragment of 99bp. Primers sequences were Fw: 5'CAC AGA AGT CTG GCC ACGT -3', and Rev: 5'- CCA ACA GAG GAC TCT TGG TCT -3'. Conditions for the amplification were the same as for ACE-1 I/D polymorphism, except hybridization at $60^{\circ} \mathrm{C}$ for 1 minute. Restriction enzyme BsI I (New England Biolabs, Beverly, Massachusetts, USA) at $55^{\circ} \mathrm{C}$ for $15 \mathrm{~min}$ was used to digest PCR products. After digestion, we have expected that $P A I-1 \quad 4 G$ allele gives $99 \mathrm{bp}$ long restriction fragment while for $P A I-15 G$ allele expected restriction fragments were $77 \mathrm{bp}$ and $22 \mathrm{bp}$ long.

\section{STATISTICAL ANALYSIS}

Statistical analyses were done with descriptive and analytical statistical methods. The significance of differences between categorical variables was tested using chi-square tests or Fisher's exact probability test, and significance between quantitative variables was tested using Student's T-test. A multivariate logistic regression model was then created in order to explore the association of outcome after three months with examined characteristics. Statistical analyses were done using SPSS 17.0.

\section{RESULTS}

This study included 94 consecutive patients with IS. Of all patients, one did not finish follow-up period of 3 months, so we recorded drop-out rate of $1.1 \%$ which is acceptable in cohort studies and could not affect the validity of our results. More than a half of patients were males $(51.1 \%)$. The average age of patients was $70.22 \pm 11.3$ years (range $18-87$ ), while the median age was 72 . The most common comorbidity was hypertension, present in 91 patients $(96.8 \%)$. The average NIHSS score at the admission was $12.5 \pm 5.0$ (range 5 $25)$, twenty four hours after thrombolytic therapy $9.9 \pm 5.7$ (range $1-25)$, and $7.8 \pm 5.9$ (range 0 - 25) at discharge. The most common complication, observed in $15(16 \%)$ patients, was haemorrhagic transformation (HT). Modified Rankin scale average score was $3.2 \pm 2.0$ (range $0-6$ ) after 3 months. Clinical and biochemical characteristics of patients, as well as cardiovascular risk factors and side effects after rt-PA therapy are given in Table 1.

NIHSS - The National Institutes of Health Stroke Scale; mRS - Modified Rankin Scale at $3^{\text {rd }}$ month after ischemic stroke; INR - international normalised ratio; APTT - activated partial thromboplastin time

The majority of patients $(44(46.8 \%))$ were heterozygotes for $4 G / 5 G P A I-1$ polymorphism and the number of homozygotes $(4 G / 4 G$ or $5 G / 5 G$ ) was equal -25 each (26.6\%). The frequencies of $4 G$ and $5 G$ alleles were the same, $50 \%$. Among the patients, 54 (57.4\%) were heterozygotes for the $A C E I / D$ polymorphism. In 9 patients $(9.6 \%) I I$ genotype was observed, and 31 patient (33\%) harboured $D D$ genotype. The frequency of $D$ allele was $61.7 \%$.

After 3 months, a favourable outcome was recorded in 26 patients $(28.0 \%)$, while the poor outcome was observed in $67(72.0 \%)$ patients, including 21 death cases (22.6\%), $13(13.8 \%)$ of those during initial hospital admission.

There was a statistically significant difference between patients with favourable and poor outcome in terms of NIHSS at admission, 24 hours after rt-PA and at discharge (Table 1). Patients with favourable and poor outcome also differed in average age (Table 1).

However, two groups (favourable and poor outcome) did not differ significantly in genotypes or alleles frequencies of $P A I-14 G / 5 G$ and $A C E I / D$ polymorphisms. The results referring to the outcomes and differences between different genotypes are shown in Table 2.

After multiple regression analysis that included effects of age, gender, NIHSS scores at admission, 24h after rt-PA and at discharge, and urea, we have observed that only age of patient (OR (95\% CI): 1.078 (1.008-1.153), $\mathrm{p}=0.028)$ and NIHSS at discharge (OR (95\% CI): 1.511 (1.0552.164), $\quad \mathrm{p}=0.024) \quad$ statistically significantly influence rt-PA therapy outcome measured by mRS.

Patients with $4 G / 4 G$ genotype had lower age of stroke occurrence $(67.38 \pm 13.83)$ compared with $4 G / 5 G$ and $5 G / 5 G$ genotypes (70.85 \pm 8.67$)$ $(\mathrm{p}=0.021)$. This difference was even more obvious when we compared $4 G / 4 G$ and $5 G / 5 G$ genotypes $(72.56 \pm 9.36)(\mathrm{p}=0.006)$. There was a statistically significant difference in the occurrence of HT between patients without $A C E D$ allele (II genotype) compared to patients with $A C E D$ allele (ID or $D D$ genotypes) $(\mathrm{p}=0.035)$ (Table 3.$)$. 
Table 1. General characteristics of patients and characteristics of patients with favourable and with poor stroke outcome after 3 months

\begin{tabular}{|c|c|c|c|c|}
\hline variable & $\begin{array}{c}\text { favourable } \\
\text { outcome }\end{array}$ & poor outcome & $\mathbf{p}$ & $\begin{array}{c}\text { all } \\
\text { n, } \% / \text { mean } \pm \text { SD, range }\end{array}$ \\
\hline Patients & $26(28.0)$ & $67(72.0)$ & & 93 \\
\hline Age & $64.69 \pm 16.39$ & $72.55 \pm 7.67$ & 0.002 & $70.22 \pm 11.3(18-87)$ \\
\hline \multicolumn{5}{|l|}{ Cardiovascular comorbidities } \\
\hline Hypertension & $24(92.3)$ & $67(100)$ & 0.076 & $91(96.8)$ \\
\hline Atrial fibrillation & $7(26.9)$ & $19(28.4)$ & 0.89 & $26(27.7)$ \\
\hline Diabetes mellitus & $6(23.1)$ & $17(25.4)$ & 0.818 & $23(24.5)$ \\
\hline Ischemic heart disease & $4(15.4)$ & $20(29.9)$ & 0.192 & $24(25.5)$ \\
\hline Heart failure & $1(3.8)$ & $11(16.4)$ & 0.168 & $12(12.8)$ \\
\hline Peripheral artery disease & $1(3.8)$ & $3(4.5)$ & 1.000 & $4(4.3)$ \\
\hline \multicolumn{5}{|l|}{ Smoking habit } \\
\hline Smokers or ex-smokers & $7(26.9)$ & $23(34.3)$ & 0.493 & $33(33.1)$ \\
\hline \multicolumn{5}{|l|}{ Personal history of diseases } \\
\hline Previous stroke & $2(7.7)$ & $7(10.4)$ & 1.000 & $9(9.7)$ \\
\hline Transitory ischemic stroke (TIA) & $2(7.7)$ & $4(6.0)$ & 0.671 & $6(6.5)$ \\
\hline Previous myocardial infarction & $1(4.0)$ & $4(6.0)$ & 1.000 & $5(5.3)$ \\
\hline NIHSS at admission & $9.96 \pm 3.63$ & $13.55 \pm 5.12$ & 0.002 & $12.5 \pm 5.0(5-25)$ \\
\hline NIHSS at $24 \mathrm{~h}$ & $5.73 \pm 2.57$ & $11.49 \pm 5.81$ & 0.000 & $9.9 \pm 5.7(1-25)$ \\
\hline NIHSS at discharge & $3.31 \pm 2.01$ & $9.51 \pm 6.00$ & 0.000 & $7.8 \pm 5.9(0-25)$ \\
\hline mRS after 3 months & & & & $3.2 \pm 2.0(0-6)$ \\
\hline \multicolumn{5}{|l|}{ Clinical parameters at admission } \\
\hline Systolic blood pressure & $157.12 \pm 26.46$ & $164.18 \pm 28.22$ & 0.274 & $161.9 \pm 27.7(100-220)$ \\
\hline Diastolic blood pressure & $86.15 \pm 12.03$ & $90.07 \pm 11.40$ & 0.146 & $88.9 \pm 11.6(60-120)$ \\
\hline Glucose level & $7.33 \pm 2.04$ & $7.69 \pm 2.73$ & 0.547 & $7.6 \pm 2.6(4.8-22.2)$ \\
\hline INR & $1.11 \pm 0.21$ & $1.14 \pm 0.16$ & 0.463 & $1.13 \pm 0.18(0.84-1.95)$ \\
\hline APTT & $26.37 \pm 4.04$ & $26.34 \pm 4.02$ & 0.978 & $26.4 \pm 4.0(19.8-38.3)$ \\
\hline Urea & $6.50 \pm 1.49$ & $7.49 \pm 2.00$ & 0.031 & $7.3 \pm 1.9(4.2-13)$ \\
\hline Creatinine & $100.13 \pm 16.10$ & $105.52 \pm 25.99$ & 0.344 & $104.1 \pm 23.8(63-194)$ \\
\hline \multicolumn{5}{|l|}{ Biochemical parameters, day 1} \\
\hline Total cholesterol & $5.11 \pm 1.46$ & $5.08 \pm 1.25$ & 0.938 & $5.1 \pm 1.3(1.5-7.5)$ \\
\hline LDL cholesterol & $4.54 \pm 0.62$ & $4.57 \pm 0.42$ & 0.906 & $4.5 \pm 0.5(3.6-5.5)$ \\
\hline HDL cholesterol & $1.45 \pm 0.27$ & $1.45 \pm 0.28$ & 0.945 & $1.4 \pm 0.3(1.1-2.0)$ \\
\hline Triglycerides & $1.55 \pm 1.01$ & $1.50 \pm 0.72$ & 0.804 & $1.5 \pm 0.8(0.4-4.8)$ \\
\hline \multicolumn{5}{|l|}{ Side effects } \\
\hline Hemorrhagic transformation & $1(3.8)$ & $14(20.9)$ & 0.059 & $15(16)$ \\
\hline Systemic bleeding & $0(0.0)$ & $3(4.5)$ & 0.557 & $3(3.2)$ \\
\hline Intrahospital infection & $4(15.4)$ & $9(13.4)$ & 0.752 & $13(13.8)$ \\
\hline Agitation & $1(3.8)$ & $4(6.0)$ & 1.000 & $5(5.3)$ \\
\hline Allergic reactions & $0(0.0)$ & $3(4.5)$ & 0.557 & $3(3.2)$ \\
\hline
\end{tabular}

NIHSS - The National Institutes of Health Stroke Scale; mRS - Modified Rankin Scale at $3{ }^{\text {rd }}$ month after ischemic stroke; INR - international normalised ratio; APTT - activated partial thromboplastin time

Table 2. Genotypes and stroke outcome

\begin{tabular}{|c|c|c|c|}
\hline & favourable outcome, n (\%) & poor outcome, n (\%) & $\mathbf{p}$ \\
\hline \multicolumn{4}{|c|}{ PAI-1 polymorphism } \\
\hline $4 \mathrm{G} / 4 \mathrm{G}$ & $9(34.6)$ & $16(23.9)$ & \multirow{3}{*}{0.530} \\
\hline $4 \mathrm{G} / 5 \mathrm{G}$ & $10(38.5)$ & $33(49.2)$ & \\
\hline $5 \mathrm{G} / 5 \mathrm{G}$ & $7(26.9)$ & $18(26.9)$ & \\
\hline \multicolumn{4}{|c|}{ ACE I/D polymorphism } \\
\hline $\mathrm{I} / \mathrm{I}$ & $0(0.0)$ & $9(13.4)$ & \multirow{3}{*}{0.100} \\
\hline $\mathrm{I} / \mathrm{D}$ & $15(57.7)$ & $38(56.7)$ & \\
\hline $\mathrm{D} / \mathrm{D}$ & $11(42.3)$ & $20(29.9)$ & \\
\hline
\end{tabular}


Table 3. Genotypes and haemorrhagic transformation

\begin{tabular}{cccc}
\hline & HT, n (\%) & without HT, n (\%) & p \\
\hline PAI-1 polymorphism & $5(5.4)$ & $20(21.5)$ & \\
4G/4G & $7(7.5)$ & $36(38.7)$ & 0.721 \\
4G/5G & $3(3.2)$ & $22(23.6)$ & \\
5G/5G & & \\
\multicolumn{1}{|c|}{} & & \\
ACE I/D polymorphism & $4(4.3)$ & $5(5.4)$ & 0.074 \\
I/I & $7(7.5)$ & $46(49.5)$ & \\
I/D & $4(4.3)$ & $27(29.0)$ & $\mathbf{0 . 0 3 5}$ \\
D/D & $4(4.3)$ & $5(5.4)$ & $5.309(1.233-22.853)$ \\
I/I & $11(11.8)$ & $73(78.5)$ & \\
I/D+D/D &
\end{tabular}

HT- haemorrhagic transformation

\section{DISCUSSION}

So far, a number of genes with products involved in processes of angiogenesis, coagulation / fibrinolysis, and inflammation were identified (21). Gene polymorphisms are responsible for different levels of gene expression or gene products activity, and they have a direct influence on cell physiology and functionality, as well as on tendencies toward specific diseases, therapy response, pathogenesis, and in the end, on the disease outcome (22). Having this in mind, in our study we have focused on the influence of the single nucleotide variant (SNP) of the PAI-I gene and the insertion/deletion variation (INDEL) of the $A C E$ gene on the recovery of the stroke patients treated with rt-PA, as well as on the incidence of haemorrhagic transformation in CNS after applying the above-mentioned therapy.

The association between $A C E \quad I / D$ polymorphism and coronary artery disease, myocardial infarction, and thrombosis was found (23). It has also been proven that the $D D$ genotype is commonly associated with the cardiovascular events (24). Apart from that, $D D$ genotype is also linked to the appearance of leucoaraiosis, in cases of lacunar infarction (25). The largest number of studies focused so far on assumption that $A C E I / D$ polymorphism may be a risk factor for the occurrence of IS. Calita and associates demonstrate that people with $D D$ genotype have a greater risk of suffering not only from IS, but haemorrhagic stroke as well (26). Scientists from China confirmed this in the meta-analysis, where they demonstrated the association between $D D$ genotype and risk of suffering an IS for Caucasian people (27). However, some other studies did not obtain the same results $(28,29)$. Malueca and associates (30) demonstrated that $D$ allele of $A C E$ $I / D$ polymorphism was associated with poorer functional recovery of patients who suffered an IS. On the other hand, very few studies have focused on the association between $A C E I / D$ polymorphism and response to rt-PA therapy after suffering an IS. Fernández-Cadenas and associates (31) focused on the association between $A C E I / D$ polymorphism genotypes and artery recanalization after rt-PA therapy on a sample of 96 IS patients. A significant link between $D D$ genotype and the extent of recanalization after $1 \mathrm{~h}, 6 \mathrm{~h}$, and $24 \mathrm{~h}$ was observed. These results do not match the ones from our study, where we could not demonstrate the association between $A C E I / D$ polymorphism genotypes and functional recovery of patients measured by $\mathrm{mRS}$ scores. Differences in findings may be consequence of different methods used for therapy efficacy assessment, since extent of recanalization may not necessarily be followed with the same extent of functional recovery.

Along with the therapy efficiency FernándezCadenas et al. also analysed the occurrence of haemorrhagic transformation based on the genotype and failed to observe the association between $A C E I / D$ genotypes and the occurrence of haemorrhagic transformation. In our study, $16 \%$ of patients had HT, and we have found a statistically significant higher occurrence of HT in patients with $I I$ genotype compared to patients with $I D$ or $D D$ genotypes $(\mathrm{p}=0.015)$. These results are contradictory to the hypothesis that $A C E D D$ genotype may be associated with intracerebral haemorrhage by impairing vasoconstriction and increasing hypertension, but this association has not been observed in Caucasian population (32). Results of our study may be explained with the findings that ACE, beside the vasoconstrictive 
effects, has a role in fibrinolysis suppression. Fibrinolysis suppression is a result of influence of angiotensin II and bradykinin, molecules regulated by ACE, on $t-P A$ and $P A I-1$ genes expression (33). Following this mechanism, lower ACE levels or activity, like in the carriers of $A C E I I$ genotype, may result in higher fibrinolysis and higher rate of HT occurrence.

In the last few years, $4 G / 5 G$ polymorphism of PAI-1 gene was often studied in the area of cerebrovascular diseases, including IS. When it comes to the association between the PAI-1 4G/5G polymorphism and IS, as well as in case of $I / D$ polymorphism of $A C E$ gene, the focus of attention was on determining the risk for different PAI-1 genotypes carriers of suffering an IS. $\mathrm{Hu}$ and associates demonstrated a significant association between the $4 G / 4 G$ genotype and increased risk of suffering an IS within an adult population, but not within children population (34). Other authors also pointed out that $4 G / 4 G$ genotype was a risk factor for suffering an IS within Chinese population (35). There are opposite results with findings that $P A I-1$ $4 G$ allele is a protective factor against IS and that it is acting through plaques stabilisation (36). To our knowledge, the only study considering association between $4 G / 5 G$ polymorphism and rtPA efficacy, conducted on 156 IS patients, reviled that $4 G / 4 G$ genotype may be responsible for the poorer recovery of patients with IS treated with rtPA (37). Patients recovery in mentioned study was measured by $\mathrm{mRS}$ and reocclusion rate. Our study could not confirm impact of $4 G / 5 G$ polymorphism genotypes on patient's recovery evaluated by modified Rankin scale, nor its influence on rate of HT. Regarding small number of available studies and complex, not fully explained role of PAI-1 activity in IS occurrence and development (36), it is still early for final conclusions.

In conclusion, our study suggests that patients with $A C E$ II genotype may be in risk of HT. This could be important finding because HT is the most feared complication of tr-PA therapy in ischemic stroke. However, additional studies on a larger number of patients are necessary in order to make this information confirmed. Our study had several limitations. The number of patients was quite small. Additionally, our survey focused only on short-term patients' outcome (after 3 months) and extra studies are necessary to explore the relationship of the SNPs to the long-term recovery and functional outcomes after stroke.

\section{ACKNOWLEDGMENTS}

This study was supported by the Ministry of Education, Science and Technological Development of the Republic of Serbia [grants no. 175091 and 175087].

\section{CONFLICTS OF INTEREST}

The authors report no conflict of interest.

\section{REFERENCES}

1. Sacco R, Kasner S, Broderick J, et al. An updated definition of stroke for the 21st century: a statement for healthcare professionals from the American Heart Association/American Stroke Association. Stroke. 2013; 44:2064-89. doi: 10.1161/STR.0b013e318296aeca.

2. Lozano R, Naghavi M, Foreman K, et al. Global and regional mortality from 235 causes of death for 20 age groups in 1990 and 2010: a systematic analysis for the Global Burden of Disease Study 2010. Lancet. 2012; 380:2095-128. doi: 10.1016/S0140-6736(12)61728-0.

3. Murray C, Vos T, Lozano R, et al. Disabilityadjusted life years (DALYs) for 291 diseases and injuries in 21 regions, 1990-2010: a systematic analysis for the Global Burden of Disease Study 2010. Lancet. 2012; 380:2197-223. doi: 10.1016/S0140-6736(12)61689-4.

4. Medic S, Beslac-Bumbasirevic L, KisicTepavcevic D, et al. Short-term and long-term stroke survival: the Belgrade prognostic study. J Clin Neurol. 2013; 9(1):14-20. doi: 10.3988/jcn.2013.9.1.14.

5. Boehme A, Esenwa C, Elkind M. Stroke Risk Factors, Genetics, and Prevention. Circ Res.2017; 120:472-495. doi: 10.1161/CIRCRESAHA.116.308398.

6. Li S, Zhao X, Wang C, et al. Risk factors for poor outcome and mortality at 3 months after the ischemic stroke in patients with atrial fibrillation. $\mathrm{J}$ Stroke Cerebrovasc Dis. 2013; 22(8):e419-25. doi: 10.1016/j.jstrokecerebrovasdis.2013.04.025.

7. Astrup J. Energy-requiring cell functions in the ischemic brain. Their critical supply and possible inhibition in protective therapy. Journal of neurosurgery. $\quad 1982$; 56:482-497. DOI: $10.3171 /$ jns.1982.56.4.0482

8. Acheampong P, Ford G. Pharmacokinetics of alteplase in the treatment of ischaemic stroke. Expert Opin Drug Metab Toxicol. 2012; 8:271-81. doi: $10.1517 / 17425255.2012 .652615$.

9. Del Zoppo GJ, Saver JL, Jauch EC, et al. American Heart Association Stroke Council. Expansion of the time window for treatment of acute ischemic stroke with intravenous tissue plasminogen activator: a science advisory from the American Heart Association/American Stroke Association. Stroke. 
2009; 40:2945-8. doi: 10.1161/STROKEAHA.109.192535.

10. Bivard A, Lin L, Parsons MW. Review of Stroke Thrombolytics. Journal of Stroke. 2013; 15(2):9098. doi: $10.5853 /$ jos.

11. Jauch E, Saver J, Adams, H, et al. Guidelines for the early management of patients with acute ischemic stroke: a guideline for healthcare professionals from the American Heart Association/American Stroke Association. Stroke. 2013; 44:870-947. doi: 10.1161/STR.0b013e318284056a.

12. Erdos E, Skidgel R. The angiotensin I-converting enzyme. Lab Invest. 1987;56:345-348.

13. Crisan D, Carr J. Angiotensin I-converting enzyme: genotype and disease associations. J Mol Diagn. 2000; 2:105-115. DOI: $10.1016 /$ S15251578(10)60624-1

14. Rigat B, Hubert C, Alhenc-Gelas F, et al. An insertion/deletion polymorphism in the angiotensin I-converting enzyme gene accounting for half the variance of serum enzyme levels. J Clin Invest. 1990; 86:1343-6.

15. Agerholm-Larsen B, Nordestgaard BG, TybjaergHansen A. ACE gene polymorphism in cardiovascular disease: meta-analyses of small and large studies in whites. ArteriosclerThromb Vasc Biol. 2000; 20:484-92.

16. Lijnen $H$. Pleiotropic functions of plasminogen activator inhibitor-1. J Thromb Haemost. 2005; 3:35-45. DOI:10.1111/j.1538-7836.2004.00827.x

17. Dawson S, Wiman B, Hamsten A, et al. The two allele sequences of a common polymorphism in the promoter of the plasminogen activator inhibitor-1 (PAI-1) gene respond differently to interleukin-1 in HepG2 cells. J Biol Chem. 1993; 268:10739-45.

18. Weisscher N, Vermeulen M, Roos YB, et al. What should be defined as good outcome in stroke trials; a modified Rankin score of $0-1$ or $0-2$ ? J Neurol. 2008; 255:867-74. doi: 10.1007/s00415-008-07968.

19. Rao NM, Levine SR, Gornbein JA, et al. Defining Clinically Relevant Cerebral Hemorrhage After Thrombolytic Therapy for Stroke: Analysis of the NINDS-tPA Trials. Stroke. 2014; 45(9):2728-33. doi: 10.1161/STROKEAHA.114.005135.

20. Miller SA, Dykes DD, Polesky HF. A simple salting out procedure for extracting DNA from human nucleated cells. Nucleic Acids Res. 1988; 16:1215.

21. Carty C, Buzková P, Fornage M, et al. Associations between incident ischemic stroke events and stroke and cardiovascular disease-related genome-wide association studies single nucleotide polymorphisms in the Population Architecture Using Genomics and Epidemiology study. Circ Cardiovasc Genet. 2012; 5:210-6. doi: 10.1161/CIRCGENETICS.111.962191.

22. Karki R, Pandya D, Elston R, et al. Defining "mutation" and "polymorphism" in the era of personal genomics. BMC Med Genomics. 2015; 8:37. doi: 10.1186/s12920-015-0115-z.

23. Danser AH, Schalekamp MA, Bax WA, et al. Angiotensin-converting enzyme in the human heart. Effect of the deletion/insertion polymorphism. Circulation. 1995; 92:1387-8.

24. Wang XL, McCredie RM, Wilcken DE. Genotype distribution of angiotensin-converting enzyme polymorphism in Australian healthy and coronary populations and relevance to myocardial infarction and coronary arterydisease. Arterioscler Thromb Vasc Biol. 1996; 16:115-119.

25. Hasan A, Lansburry A, Catto AJ, et al. Angiotensin converting enzyme insertion/deletion is associated with leukoraiosis in lacunar syndromes. J Neurol Neurosurg Psychiatry. 2002; 72:343-6.

26. Kalita J, Somarajan B, Kumar B, et al. A study of $\mathrm{ACE}$ and ADD1 polymorphism in ischemic and hemorrhagic stroke. Clin Chim Acta. 2011; 412:642-6. doi: 10.1016/j.cca.2010.12.022.

27. Yuan H, Wang X, Xia Q, et al. Angiotensin converting enzyme (I/D) gene polymorphism contributes to ischemic stroke risk in Caucasian individuals: A meta-analysis based on 22 casecontrol studies. Int J Neurosci. 2015; 1-24. doi: 10.3109/00207454.2015.1036421.

28. Tuncer N, Tuglular S, Kilic G, et al. Evaluation of the angiotensin-converting enzyme insertion/deletion polymorphism and the risk of ischaemic stroke. J Clin Neurosci. 2006; 13:224-7. DOI: 10.1016/j.jocn.2005.08.005

29. Domingues-Montanari S, Fernandez-Cadenas I, del Rio-Espinola A, et al. The I/D polymorphism of the ACE1 gene is not associated with ischaemic stroke in Spanish individuals. Eur J Neurol. 2010; 17:1390-2. doi: 10.1111/j.14681331.2010.03022.x.

30. Malueka RG, Dwianingsih EK, Sutarni S, et al. The $\mathrm{D}$ allele of the angiotensin-converting enzyme (ACE) insertion/deletion (I/D) polymorphism is associated with worse functional outcome of ischaemic stroke. Int J Neurosci. 2017; 1-8. doi: 10.1080/00207454.2017.1412962

31. Fernández-Cadenas I, Molina CA, Alvarez-Sabín J, et al. ACE gene polymorphisms influence t-PAinduced brain vessel reopening following ischemic stroke. Neurosci Lett. 2006; 398:167-71. DOI:10.1016/j.neulet.2005.12.079

32. Sun Y, Liu Y, Watts LT, et al. Genetic associations of angiotensin-converting enzyme with primary intracerebral hemorrhage: a meta-analysis. PLoS One. 2013; 8(6):e67402. doi: 10.1371/journal.pone.0067402

33. Douglas E. Vaughan. Angiotensin, Fibrinolysis, and Vascular Homeostasis. Am J Cardiol. 2001; $87: 18 \mathrm{C}-24 \mathrm{C}$

34. Hu X, Zan X, Xie Z, et al. Association Between Plasminogen Activator Inhibitor-1 Genetic Polymorphisms and Stroke Susceptibility. Mol Neurobiol. 2017; 328-341. DOI:10.1007/s12035015-9549-8 
35. Cao Y, Chen W, Qian Y, et al. Plasminogen activator inhibitor-1 $4 \mathrm{G} / 5 \mathrm{G}$ polymorphism and ischemic stroke risk: a meta-analysis in Chinese population. Int J Neurosci. 2014; 124:874-81. doi: 10.3109/00207454.2014.886577

36. Hoekstra T, Geleijnse MJ, Kluft C, et al. 4G/4G genotype of PAI-1 gene is associated with reduced risk of stroke in elderly. Stroke. 2003; 34(12):28228.

37. Fernandez-Cadenas I, Del Rio-Espinola A, Rubiera $\mathrm{M}$, et al. PAI-1 4G/5G polymorphism is associated with brain vessel reocclusion after successful fibrinolytic therapy in ischemic stroke patients. Int J Neurosci. 2010; 120:245-51. DOI: $10.3109 / 00207451003597169$ 